\title{
La versión lega de sabiduría y sabio en el refranero español: un acercamiento complementario a los estudios al uso
}

\author{
Vicente Pelechano \\ Universidad de La Laguna (Tenerife, España)
}

\section{RESUMEN}

Se propone la utilización y significación de los refranes como recurso para conocer los modelos legos de sabiduría, junto a los procedimientos de calificación de adjetivos y de nominación de personas o actos sabios. La sabiduría, en la filosofía popular (i) se encuentra significativamente cercana a la idea de inteligencia; (ii) posee un fuerte componente ético; (iii) exige no solamente cognición sino asimismo acción; (iv) reconoce la importancia de recibir y emitir buenos consejos procedentes tanto de hombres como de mujeres; (v) contrapone sabiduría con necedad y percibe el mundo sabio y el necio como incompatibles en convivencia aunque se admite que actos necios los hacen tanto los sabios como los necios; (vi) la sabiduría se aprende con esfuerzo y contactos frecuentes con personas sabias, por lo que no es cuestión de herencia; (vii) el saber más difícil de alcanzar es el que se refiere a uno/a mismo/a; (viii) el mundo de la mujer se restringe en gran medida al hogar, pero en él, ocupa el lugar central tanto en decisiones como en acciones y concepciones acerca de lo que son las relaciones interpersonales y su dirección; (ix) sabiduría y riqueza no se cultivan a la par; $(x)$ el sabio se presenta con algunos atributos negativos como el temor, la imposibilidad de amar y la avaricia y psicopatología (xi) la sabiduría tendría como objetivo el crecimiento personal, pero no el logro de la felicidad ni de la riqueza.

Palabras clave: refranes y teoría lega; teorías implícitas de sabiduría; conocimiento tácito en sabiduría; los refranes como depósitos de sabiduría; teorías implícitas y explícitas de sabiduría.

\section{ABSTRACT}

The analysis of sayings it is proposed as recourse in the study of lay theories of wisdom. In Spanish sayings, (i) wisdom is very near to the meaning of intelligence for adult people; (ii) this knowledge has a strong ethic component; (iii) wisdom mean a cognitive psychological attribute which it demands also action; (iv) it is important to ask and to give advices; (v) wisdom and foolishness are incompatible, but inclusive wise people makes foolish acts; (vi) wisdom it is not hereditary. Wisdom is learned through effort and hard work in collaboration with wise people; (vii) the wisdom more difficult is the personal wisdom about one-self; (viii) the women have a central place at home and are considered specialized in the wisdom of personal relationships; (ix) wisdom and wealth are not compatible; $(x)$ the wise is characterized with some negative attributes such as miserliness, fear, impossibility to love and some psychopathology and (xi) the aim of become wise it is the personal growth, not to be happy.

Keywords: Sayings and lay theory; implicit theories of wisdom; tacit knowledge in wisdom; sayings as wisdom's store; implicit-explicit theories of wisdom as a procedure more. 


\section{Una nota sobre acercamientos legos y académicos}

Aunque resulta prácticamente imposible datar el arranque en el estudio de un tema, parece que, según informaciones procedentes de la propia autora, Vivian Clayton es una protagonista principal, aunque no la única, de la incorporación del estudio de la sabiduría en psicología, en la década de los setenta y precisamente al plantearse el tema de su tesis doctoral. Hasta ese momento, C. G. Jung, desde presupuestos de psicología profunda había señalado que la sabiduría era un elemento importante en el funcionamiento de los grupos humanos y los ancianos venían a encarnar una parte del conocimiento del inconsciente colectivo del grupo y, desde aquí, representarían un punto de referencia a la hora de tomar decisiones graves acerca de la pervivencia del grupo. Un poco después, Erikson $(1959,1986)$ propuso en su teoría evolutiva del ciclo vital, ocho fases, las tres últimas dentro de la fase adulta; una última fase que, si se resolvía de manera satisfactoria, se daría en la fase anciana (integridad frente a desesperanza: integridad del yo frente a desesperanza que representaría la aceptación del papel necesario de la muerte en la renovación constante del ciclo de la vida, con la virtud resultante que sería la sabiduría ) y tendría como resultado el logro de sabiduría (adquisición de una intuición especial sobre la significación de sus vidas y de la existencia en general, a la que se daría un sentido de continuidad para enfrentarse a la muerte con dignidad). En ambos casos se identificaba el alcance de sabiduría con la fase anciana por un lado $y$, por otro, era la antesala de la muerte (claro que la muerte llegaba habiendo alcanzado la sabiduría o no; pero el caso es que se asociaba la sabiduría con la fase del fin de la vida, con lo que su estudio se asociaba, de una u otra forma, con la cercanía de la muerte, lo que no resultaba especialmente atractivo).

La evolución en el estudio empírico de la sabiduría ha tomado dos caminos a partir de su conceptualización primera como conocimiento pragmático y capacidad para solucionar o aliviar los problemas de la vida, que llevaría consigo tanto la lógica bivariable típica de la ciencia natural, como la lógica dialéctica que se mueve entre disonancias y contradicciones (tal y como lo hace la propia vida): el primero ha sido el de estudiar la imagen y/o el modelo que las personas no especialistas poseen de sabio y de sabiduría (acercamientos denominados legos o de "sentido común") y, en contraposición a esta opción, el estudio "científico" y académico de la sabiduría. En este trabajo nos ocupamos principalmente del acercamiento lego, puesto que del científico nos hemos ocupado en otro lugar (Pelechano y González, 2015).

Ante el panorama desolador en cuanto a existencia de resultados empíricos, Clayton ofreció una alternativa (Clayton y Birren, 1978): anclar el concepto de "sabiduría" en relación con otros conceptos psicológicos, que podrían estar relacionados con ella, tales como "anciano", "inteligente", "erudito", "observador", "agradable", "cordial" hasta 15 descriptores y se pidió a los participantes adultos que compararan todos los descriptores entre sí y con relación a "sabio", "anciano" y "yo mismo". El estudio de las calificaciones respecto a similitud de esos términos mostraron tres conglomerados de 
atributos a los que denominaron dimensiones de características cognitivas, afectivas y reflexivas de las personas sabias. En un estudio similar, Brent y Watson (1980) pidieron a los participantes que describieran las características de una persona sabia y, en esta ocasión, se aislaron cuatro conglomerados que denominaron personal-cognitivo, práctico-experiencial, interpersonal y ético-moral. En el trabajo, de este período, posiblemente más completo en este campo, Holliday y Chandler (1986) asentado en la teoría de la categorización de Rosch (1978) llevaron a cabo tres estudios con el objetivo de aislar las dimensiones que definirían la sabiduría y además, en tres muestras de edad (medias de 22,2; 43,4 y 69,88 años). El análisis factorial (componentes principales) de los descriptores (79, sobre una muestra de 150 adultos) aisló cinco factores que se denominaron: (i) comprensión excepcional (ejemplos de atributos: sentido común, aprende de la experiencia, intuitivo); (ii) habilidades de enjuiciamiento y comunicación, como ser consciente, es una fuente de buen consejo, comprensivo, entiende de la vida, valora atender a la información que recibe, reflexivo, piensa antes de decidir; (iii) competencias generales como curioso, pensativo, comprende y evalúa la información, lector, inteligente, estructurado; (iv) habilidades interpersonales, como agradable, sensible, fiable, escucha con atención, amable, sociable, moral, paciente, altruista, espiritual, feliz, maduro y (v) facilitador social (no perturbador) como discreto, tranquilo, planificador y cuidadoso. En un tono similar de atributos positivos se expresan Brent y Watson (1980) y, en un trabajo que es bastante complejo, Stern- berg (1985) se mueve en estos términos, si bien señalando unas diferencias en los atributos en función de la especialización profesional de los calificadores (profesores universitarios de distintas especialidades).

Otro tipo de estudios ha consistido en la petición a los participantes para que nombraran a personas que consideraran que habían sido o eran sabias (Denney et al., 1995; Jason et al., 2001; Paulhus, Harms y Strausser, 2002; Pelechano y González, 2004, 2008; Perlmutter, 1988). Los resultados alcanzados tienden a corroborar que cuando se habla de sabiduría y de sabios todos los calificativos utilizados se refieren a atributos positivos, coherentes con la idea de que la sabiduría es una de las más admirables excelencias humanas, y el sabio estaría caracterizado preferiblemente por ser mayor, ser hombre (se citan a muy pocas mujeres), tienden a ser líderes políticos o religiosos, que se ocupa en la solución de problemas de orden social e interpersonal y que es poco frecuente su presencia entre los seres humanos (Pelechano y González, 2015). En el caso de los adolescentes, la sabiduría no se considera similar ni cercana a la inteligencia, aunque estos dos conceptos se consideran semánticamente cercanos en el caso de los adultos.

Frente a esta manera de estudiar la sabiduría se encuentra la que corresponde a la "ciencia" o a la academia. Contemporáneamente con estos estudios, P. Baltes en Alemania comenzó el estudio de la sabiduría desde presupuestos asentados en la teoría de Cattell sobre inteligencia fluida y cristalizada. Baltes (19392006) entiende la sabiduría como un atributo psicológico de excelencia que es fundamen- 
talmente cognitivo, se ocupa de los problemas relevantes de la vida y que se mantiene hasta cerca de los 90 años de vida. Ha ofrecido un conjunto de cuatro criterios para su evaluación, lo que lleva consigo un modelo de evaluación y ha llegado a compilar el mayor volumen de datos hasta comienzos de este siglo. En los estudios realizados se presentaba a cada participante un problema ficticio y se le pedía a cada participante que dijera todo lo que se le ocurriera para resolverlo; las respuestas se grababan y se pasaba después al análisis de las mismas según los criterios de corrección. El primer criterio es el conocimiento de hechos, y se refiere al conocimiento (el qué se conoce) que se posea en el dominio al que pertenece el problema; el segundo es el conocimiento de los procedimientos (el cómo) adecuados para hallar la solución; el tercero es la contextuación de las informaciones que se manejen para resolver el problema en los dominios de la vida y el cuarto es la existencia de un cierto relativismo de valores propios en comparación con los de los de los demás, aunque de ninguna manera se defiende un relativismo axiológico total (en el sentido de que "vale todo"). Alternativamente Pelechano y González (2015) han ofrecido el modelo SAMUL de sabidurías múltiples en donde se postulan siete tipos de sabiduría, con instrumentos de evaluación, resultados y relaciones con personalidad. Entre ambos acercamientos, dos escalas de sabiduría: una, la de Ardelt (2003) que es una versión ampliada en forma de cuestionario de los tres atributos que defendió Clayton a comienzos de los 80 y la otra, la de Webster (2003) más fina metodológica- mente y con cinco factores aislados por procedimientos de análisis factorial.

El caso es que en los dos acercamientos (el lego y el académico) se identifica a la sabiduría y al sabio solamente con atributos positivos y de ahí una de las derivaciones actuales de ciertos autores como Staudinger (2011), Ardelt (2008) y Bassett (2011?) de identificar la personalidad del sabio como una personalidad "madura" que lo acercaría al "santo" o, alternativamente, Sternberg (2013) que lo identificaría como una personalidad "altruista" en la medida en que junto a una inteligencia práctica debe existir un equilibrio en las acciones sabias entre los intereses intrapersonales, extrapersonales e interpersonales y el propio Baltes ha estado insistiendo hasta su muerte en el reconocimiento de elementos de bondad ética en su concepción (como sucedía con Aristóteles en el siglo IV antes de Cristo, la prudencia se considera, en la actualidad, como una virtud o un tipo de sabiduría, la sabiduría personal).

Es posible que este camino sea el correcto, aunque también es posible que la intensificación hacia los atributos y elementos positivos del funcionamiento personal humano sea un sesgo explicable por el cultivo de la "psicología positiva", por el material utilizado para el estudio de la sabiduría hasta nuestros días, incluso, simplemente por el sesgo en la elección de las expresiones a calificar o por el componente ético que presenta cierta acepción de la sabiduría presente en gran parte de la tradición filosófica. Y no deja de llamar la atención el hecho de que tanto en las tradiciones orientales (confucionismo y budismo, por ejemplo) como en las occidentales (filosofía occidental en sus distintas 
versiones), se apele a la necesidad de exigencias éticas dentro del pensamiento de sabiduría. Sucede, además, que en la medida en que se justifique el cultivo de la sabiduría en el ser humano el objetivo es del logro de la felicidad, lo que se adquiere, según las distintas tradiciones, a partir de un tipo de conocimiento al que se acompaña de la práctica de la virtud.

\section{Una opción lega alternativa: los refranes como recurso}

Desde hace ya más de una década venimos reconociendo el enorme cuerpo de refranes españoles como material que merecería una atención especial a la hora de la elaboración incluso, de instrumentos y fruto de ello han sido dos cuestionarios y dos pruebas de rendimiento máximo: (a) uno acerca de la "personalidad cultural" española; (b) una escala de prudencia y sensatez; (c) un test de rendimiento máximo en el que se pide la definición de unos refranes y tres ejemplos de aplicación correcta de cada refrán al mundo social y (d) un test compuesto por 24 pares de refranes aparentemente contradictorios y en donde se pide al sujeto el reconocimiento de la contradicción o la "salida" dialéctica ante esa contradicción aparente.

Un refrán es una expresión más bien corta, que es o ha sido de utilización frecuente, fruto de la experiencia y el ingenio, que se hizo o se ha hecho célebre por ser aguda, de origen antiguo y que posee una considerable variedad de denominaciones y/o sinónimos tales como proverbio, aforismo, apotegma, adagio, sentencia, decir, dicho, cantar, ensiemplo, castigo o castigamiento (utilizado como corrección o enseñanza), fabliella (por fábulas o moralejas), brocárdico (del francés brocard, pulla o dicho chistoso), aforismo de jurisprudencia, evangelio chico o abreviado, filosofía vulgar e incluso sabiduría de las naciones y que proviene, al parecer, del francés "refrain" (recogida con la significación de estribillo en las Siete Partidas de Alfonso X el Sabio, en el siglo XIII y en el Libro de las armas del infante Don Juan Manuel, en el siglo siguiente; de la expresión utilizada ya en el siglo XIV por el Arcipreste de Hita:"retráeres", o en los "Refranes que dicen las viejas tras el fuego" del Canciller Pedro López de Ayala en 1499. Los refranes se refieren a prácticamente todos los hechos y sentimientos humanos, proceden básicamente del habla popular aunque las diversas plasmaciones han sido hechas por autores tales como Cervantes, el padre Isla y Moreto, lingüistas como Covarrubias, Rodriguez Marín y, una de las últimas, la compilación de 64.054 refranes, además, ordenados, hecha por Martínez Kleiser (1989).

A partir de aquí, la sugerencia, de la que este trabajo representa una muestra, de rastrear la significación de "sabio" y "sabiduría" que se encuentra presente en el refranero español, intentando ofrecer una imagen más o menos coherente de lo que la psicología popular y culturalmente decantada tiene de estas expresiones, que no siempre es coherente con lo que dice la bibliografía al respecto. Se han utilizado las compilaciones más reconocidas por la Real Academia, básicamente las de Gonzalo Correas (1627/1992), Irribarren (1994), Martínez Kleiser (1989), Rodriguez Marin (1926) y Sbarbi (1891/1980) y la revisión se ha centrado en elementos comprometidos con "conocimiento", "consejo", "inteligencia/ 
inteligente", "prudencia" y "sabiduría/saber". Se han eliminado las redundancias (cuando el mismo refrán aparece en más de un lugar) y las variaciones de una misma idea (en más de una ocasión, un refrán se expone con varias expresiones, en cuyo caso el autor ha elegido aquella más eufónica).

Los refranes se han utilizado en diversas ocasiones en psicología, a veces como elementos de prueba de inteligencia y en otras ocasiones, en las que se ha evaluado la lesión de determinadas zonas corticales. Y, a la vez, han sido objeto de discusión en la medida en que se les acusa de ser contradictorios. El caso es que, por lo que se refiere a sabiduría, se trata de un tipo de conocimiento que lleva consigo ambigüedad e incluso contradicción, elementos que deben ser analizados y, en más de una ocasión, resueltos por la persona que tiene que resolver uno de estos tipos de problemas.

La difusión de su utilización, así como su origen y larga duración de uso ha propiciado que (a) se haya tergiversado su forma, significado y extensión como "Quien calla, otorga”, del original "Quien calla, no dice nada"; o "Un clavo saca a otro clavo" del original "Un clavo saca a otro clavo, si los dos no quedan dentro"; (b) también se da el caso de que se haya perdido por el uso (mal uso) una parte del refrán como en los casos “Un buen paño en el arca se vende ( $y$ falta: mas el malo verse quiere"), "Quien tiene boca se equivoca" ( $y$ falta: "pero quien tiene seso, no dice eso"); (c) asimismo se encuentran expresiones que se complementan una a la otra como "A gran cabeza, gran talento (si es que lo tiene dentro"), "Cobra buena fama y échate a dormir (y mira no te duermas, porque no la pierdas)" o "Por dinero baila el perro (no por el son que toca el ciego"); (d) y también se dan contradicciones aparentes como "Querer y poder, hermanos vienen a ser" y "Querer y poder, no es todo uno", contradicción que desaparece porque dos hermanos, aunque hermanos, no son una misma persona, por lo que hay diferencias entre ellos. $\mathrm{O}$, “Una espiga no hace manojo", que defendería un desprendimiento frente a "Una gota mengua la bota" que llama al ahorro y que ambas pueden coexistir sin quebranto; (e) en otras ocasiones, las aparentes contradicciones son fruto de un mal aprendizaje de los refranes, que se cambian al repetirlos con el uso continuado, contagiándose de mala utilización que no siempre carece de sentido como "Más quiero ser de vieja rogada, que de moza desdeñada" que se convirtió en "Más quiero ser de moza rogada que de vieja desdeñada" (que defiende lo contrario al caso anterior); (f) o "Pasión no quita conocimiento" frente a "Pasión nubla conocimiento", que representan dos significaciones paralelas al centrarse en segundo en que la pasión puede cegar el entendimiento, mientras el primero es más bien la expresión elíptica defectuosa de un deseo.

Sirva lo dicho para mostrar por un lado la versatilidad, por otro la complejidad y extensión de dominios en los que se utilizan refranes, que llegan hasta la receta culinaria y su consumición como "Para hacer una buena ensalada, cuatro hombres hacen falta: para la sal, un sabio; para el aceite, un pródigo; para el vinagre, un avariento y para revolverla, un loco; llega luego un hambriento, y se come en un dos por tres lo que hicieron el sabio, el pródi- 
go, el loco y el avariento", sin olvidar los refranes "de la temperie" o meteorológicos.

\section{Inteligencia y sabiduría}

Los refranes han sido empleados, mayoritariamente, por adultos $y$, en esta fase de evolución (Pelechano y González, 2004, 2006), inteligencia y sabiduría se tienden a percibir como dos dominios tan cercanos que, a veces, se confunden, aunque no en el período de adolescencia. En el refranero no se insiste en las diferencias entre ambos términos, aunque podrían encontrarse ejemplos de ambas expresiones (inteligencia y saber solapándose con talento), aunque en todos los casos, el refranero no toma en consideración un acercamiento de ciclo vital sino que se suele restringir a adultos, y en todo caso, a ancianos.

(1). Se valora la inteligencia y el saber.- Se entiende inteligencia en el refranero, no en un sentido psicométrico, sino como capacidad para resolver problemas de todo tipo y se valora muy positivamente ("Un hombre con buen talento, vale por ciento"), aunque parece claro que no se reconocen usualmente las limitaciones que cada uno tiene en esa capacidad ("Si cada uno se juzgase, no sería menester alcalde"; o "De su talento, ninguno hay descontento"), todo el mundo tiene suficiente y este suficiente siempre se entiende como gran capacidad. Y una sugerencia que puede representar un reto a la hora de diseñar una sociedad no competitiva en cuanto a poder físico, económico o mental: "Cada uno mida con su palmo, y así ni el gigante es gigante, ni el enano es enano" lo que habla tanto de realismo en la estimación de las propias posibilidades, como en el reconocimiento implícito de que existen distintos tipos, intensidades o magnitudes de inteligencia. Estas diferencias aparecen con más claridad en los diseños inter-sujeto que en los intra-sujetos si éstos son de períodos cortos.

Es la inteligencia (el entendimiento) lo que diferencia a los seres humanos de los animales ("Por el entendimiento se distingue al hombre del jumento"). Este conocimiento debe tener limitaciones que debe poner el propio individuo porque "El mucho saber, echa al hombre a perder", lo que exige alguna explicación. Y esta explicación se orienta hacia la detección de las insuficiencias que un acercamiento exclusivamente cognitivo posee para la vida plena y eficaz: el mero conocimiento que se tenga de los hechos y fenómenos es insuficiente, si no va acompañado de buena conciencia (intenciones y moral) como se reconoce en "Mucha ciencia es locura, si el buen seso no la cura", por lo cual "Mucha ciencia y poca conciencia, mala ciencia". Y entre esa "conciencia" se encuentra la supresión de la ambición: "A quien lo bastante tiene y no quiere más, por sabio lo tendrás".

La prudencia aparece en el contexto de la inteligencia. Como guía de la acción inteligente: "De nada sirve la ciencia si no gobierna la prudencia", lo que representa ya desde aquí, una llamada de atención acerca del objetivo del bien social que es la educación: no se trata solamente de adquirir conocimientos sino de su utilización.

(2). Valor de la herencia para las capacidades intelectuales.- Se habla en el refranero del valor de la "naturaleza" que vendría a ser un trasunto de la herencia biológica pues "Sin buen natural, no hay ciencia que valga", o "Lo que la 
cuna da, solo el ataúd lo quitará", que representa un límite al valor del aprendizaje como en "Más vale puñado de natural que almoradas de ciencia".

(3). El aprendizaje del saber.- La sabiduría se aprende y ello exige dedicación, motivación y esfuerzo ("Del tiempo y la experiencia es hija la prudencia"; "Ninguno se hace sabio sin trabajo"), porque la sabiduría no se hereda ("Nadie es sabio por lo que supo su padre", "En la cuna, sabiduría ninguna" o "El tonto nace, el sabio se hace"). Y este aprendizaje se predica con la palabra ("Platicar con un hombre muy instruido, es leer a la vez muchos libros"), el diálogo con los sabios ("Entre todos sabemos casi todo", o "Todo el saber no cabe en una cabeza, por buena que sea" $y$ "Todos los hombres lo saben todo, más no uno solo" o "Quien con sabios entiende, a ser sabio aprende") y la acción.

El proceso de adquisición del saber lleva consigo inseguridades e incluso temores: "Al saber y al dudar, siempre juntos los vi andar" $y$ con prudencia otra vez ("Nunca atices el fuego con la espada"), y trabajo duro ("Trabajos hacen al hombre sabio"), en un período temporal largo ("En pocos días no cabe gran sabiduría").

Debe tenerse en cuenta, con todo, la paradoja del saber que se sabe más cuando se sabe menos: "A más saber, más nos queda por aprender" (o "Solo sé que no sé nada"), y de forma explícita:"El primer año, doctor; el segundo licenciado; el tercero bachiller; el cuarto estudiante; el quinto ignorante que comienza y quiere saber", frase que se atribuye asimismo a Confucio. Y, a la vez se va haciendo sabio "Poco a poco, en tiempo largo, el ignorante se hace sabio". No se trata de ninguna contradicción sino la apelación a la infinitud posible del saber y la finitud de la propia vida a ello dedicada.

\section{La sabiduría es más que un atributo cognitivo}

La sabiduría exige no solamente leer y pensar: "Para saber, no basta leer: hay que andar y ver" o "No presuma de tener ciencia quien no tiene experiencia", $y$, además, actuar: "Sabio se ha de llamar al que sabe bien obrar", "Aquel es sabio que obra como sabio" porque de otra manera puede ser "Necio por natura, sabio por lectura", que viene a ser una intensidad aminorada de saber, reforzada por "No presuma de tener ciencia, quien no tiene experiencia".

$Y$ dentro de lo que representa conocer, el refranero distingue lo que es conocer de manera más y menos profunda, como "Saber mucho y tener mucho saber, una cosa parecen y dos vienen a ser" o "Sabio no es el que más sabe sino el que mejor lo sabe". Sin autoengaños de consolación porque "Muchos piensan llegar a la ciudad de la sabiduría y se estancan en la venta de la pedantería", "A veces el sabio, necio parece".

\section{El valor de consejos y consejero}

La sabiduría tiene dos funciones principales: una es la solución directa de los problemas, la otra, es la emisión de consejos que puedan aliviar la situación problemática que se esté planteando. $Y$ el aconsejar forma parte asimismo de muchos procedimientos terapéuticos. Sin embargo, no todos los consejos valen, ni valen de la misma manera. Estando claro que estos consejos no tienen grado de verdad perfecto ("Consejos ciertos, los que a 
los vivos, dan los muertos"), debe comenzarse por prestar mucha atención en la elección del consejero porque "Ten a todos por amigos y a uno por consejero", o "Quien buen consejero tuvo y lo desoyó, su mal buscó". Si hay alguien que busca consejo hay que oir pero no decidir de entrada ofrecerle el consejo que uno cree porque "Quien consulta, aprobación busca" y a veces el consejo no se la da.

Debe fijarse en el contenido del consejo más que en quien lo emite porque aunque "Cada necio quiere dar su consejo", "De un hombre necio, a veces, buen consejo". Una estrategia conservadora más frecuente: "El consejo, pídelo al sabio y no al necio" (o "Necio es y más que necio, quien a necios pide consejo").

Como un primer criterio de selección acerca de la fuente del consejo: "El mejor consejero, el que no busca dinero", o "El buen consejero, se aconseja a sí primero" y por supuesto, "Quien ha de aconsejar, ha de saber más que ha de hablar", siendo consciente de que "Hecho el hecho, huelga el consejo" (o "Consejo tardío, consejo baldío").

Debe hacerse caso de los consejos porque la decisión última es de cada cual y no del emisor el consejo: "Aconsejar no es mandar". Un buen consejo es saludable: "Llévate del buen consejo, serás sabio y morirás viejo" (o "Quien no oye consejo, no llega a viejo"), "En el más viejo está el buen consejo".

Aunque el consejo debe ser pedido y no dado como hábito o costumbre porque "Mucho aconsejar no suele agradar", "El consejo no es bien recibido, donde no es pedido" $y$ por ello, "Quien da consejo no pedido, pierde el consejo y el amigo".
El consejo posee un valor limitado: "El que aconseja no paga más que con buenas palabras" $y$ "Bien predica el ayunar, quien acaba de almorzar". Porque "Del sabio, el consejo, del rico, el remedio".

\section{El objetivo de la sabiduría: crecimiento personal}

Valga como ejemplo: "Saber por solo saber, cosa vana viene a ser; saber para ser mejor, eso es digno de loor" o "Quien no sabe aprovecharse de lo que sabe, poco sabe". La dedicación al saber con exclusión de todo lo demás tampoco es aconsejable porque primero es vivir: "Más vale burro vivo que sabio muerto" y, alternativamente "Más vale perro vivo que león muerto").

\section{Sabiduría y necedad}

Deberían ser considerados los dos extremos de muchos ejes que interactúan entre sí entre los cuales podrían ejemplificarse los siguientes:

1. La necedad como una mala aplicación del saber, como en "No hay tontería que no esté apadrinada por la sabiduría", "A veces el sabio, necio parece", que puede tener alguna ventaja si el sabio se encuentra interactuando entre necios ("Con el necio serás más, y con el sabio sabrás"), "Antes que se le ocurra una necedad a un mentecato, se le ocurrió a un sabio", o"Tontos sabios, nunca ví, pero sabios tontos, sí".

La sabiduría, por otra parte, no es una vacuna en contra de la necedad en la medida en que "El mucho saber no quita el mal hacer". Y debe tenerse cierto cuidado en la acción dado que"El sabio piensa las cosas, y el loco las hace". 
2. La necedad como ausencia de sabiduría por naturaleza, unida muchas veces con intolerancia de ambigüedad e impulsividad, como "Tonto instruido, tonto perdido", "El tonto nace, el sabio se hace", y que resulta molesta la convivencia entre sabios y necios: "Al sabio le es molesto hablar entre necios", "El sabio enfada si entre necios habla", "El burro empinado, tiénese por sabio" y "El sabio que es tonto, da la cara pronto".

3. Se reconoce que la falta de sabiduría si va acompañada de suerte (ventura) permite una vida alternativa a la que propone la sabiduría porque la sabiduría no lleva a la riqueza: "Con ventura y sin saber, rico llegarás a ser; con saber y sin ventura, pobre hasta la sepultura", idea, la de la pobreza económica unida al conocimiento que se repite como: "El mucho saber, cuando tuvo que almorzar, no tuvo qué comer", o “Sabio sois? Pobre moriréis" y "Saber sin haber, poco suele valer". Refranes que recogerían la tradición española de devaluación del saber, que al parecer, ya estaba presente desde fines del siglo XV.

4. Sabios y necios no se comunican de manera satisfactoria.- En el refranero se trata de dos tipos de seres humanos que son incompatibles: "Al sabio le es molesto hablar entre necios", "El sabio enfada, si entre necios habla". La sociedad no promueve el saber sino la necedad: "El sabio es menospreciado, y el necio rico, estimado" y "Cuando el sabio yerra, más que al necio le hacen la guerra". Aunque existen humanos entre los dos grupos extremos que pueden convivir conscientes de su capacidad: "Con el necio serás más, y con el sabio sabrás", porque "Sabios conocí, sabios para los otros y necios para sí" o "Nada sabe quien lo que le importa no sabe".

5. Sabio y sabiduría son incompatibles con la presunción y los avatares de la vida.- El sabio no presume de su sabiduría: "A quien presume de sabio, por necio hay que dejarlo", o "Mientras más tu saber alabes, menos sabes". El sabio no resulta alterado por los sucesos de la vida: "Al sabio, ni el bien lo aloca, ni el mal lo apoca".

\section{El conocimiento de sí mismo/a}

Resulta una tarea de gran dificultad: "Harto difícil es conocerse uno a sí mismo, porque cada persona es un abismo" pero muy importante: "No hay cosa de más saber, que a sí mesmo conocer" o "Vemos menos lo que está más cerca". Existen muchas variaciones de este tema desde "Bien ve el ciego cuando se mira por dentro", "Mírate a ti mismo y entrarás en un abismo", "Antes de decir de otro "cojo es", mírate los pies". Y a esto se acompaña la conveniencia de tomar en cuenta a los demás: "No hay mejor espejo que los ojos ajenos", o "Entre la del amigo que te alaba y el enemigo que te vitupera, está tu calificación verdadera" y "Quien a otro quiere juzgar, por sí debe comenzar".

\section{Sabiduría y sentimientos}

Tener sabiduría se percibe incompatible con el amor y en este punto no parecen haber fisuras: "Mucho saber y mucho amar, no pueden juntos estar" o "Cuando más se sabe, menos se siente".

\section{Atributos negativos y psicopatología}

Existen aspectos negativos en el saber porque saber, como tal, no es una vacuna contra 
la maldad y así "El mucho saber no quita el mal hacer" o"Sabio y malo es muy peor que bueno e ignorante".

Saber puede ir aparejado de mala suerte, pero el sabio se resigna con ella. "Al sabio su suerte le agrada, aunque sea mala". Puede llegar a ser impulsivo ("El sabio que es tonto, da la cara pronto") y temeroso ("El sabio teme, el necio se atreve").

Y, frente a la afirmación de que la sabiduría podría cultivarse para alcanzar la felicidad, el refranero es taxativo: "Mucho saber hace sabios, pero no dichosos" $y$, de forma más rotunda: "Mucho saber trae padecer".

Se atribuye avaricia a la sabiduría: "Sabios, casi siempre avaros", o "Sabios, según la experiencia, más avarientos de oro que de ciencia".

La sabiduría suele ir aparejada, además, de locura: "A veces, el sabio se hace el loco y aún lo es un poco", o "No es sabio del todo quien no tiene algo de loco" $y$, en todo caso se apunta una diferencia: "El sabio piensa las cosas, y el loco las hace".

\section{Envejecimiento y sabiduría}

Sobre este punto existe una abundancia de refranes:"En las barbas no consiste el saber, sino en el mucho estudiar y en el mucho ver", "Canas son vejez, que no saber", "Hombre cano, viejo, más no sabio", "Saber no va en canas, ni valor en barbas". Con el alargamiento de la vida y la concomitancia de envejecimiento con enfermedades, los refranes aparecen como especialmente adecuados en la mayoría de los casos.

\section{Unas notas acerca de la ubicación de la mujer}

En el refranero se dedica una extensión considerable a funciones e identidades de la mujer. Por lo que se refiere a sabiduría se previene porque la mujer puede ser más sabia que el hombre, en cuyo caso "No te cases con mujer que te gane en el saber". A partir de aquí y por lo que se refiere a la sabiduría podrían hacerse algunas observaciones.

De entrada no se desea a la mujer culta: "Ni joya prestada, ni mujer letrada" porque "Mujeres y libros, siempre mal avenidos". Aunque existen mujeres sabias y/o inteligentes: "Mujeres hermosas y con talento, una de ciento; y si mejor lo he de decir, una entre mil". Un problema que se presenta en las mujeres es el mantenimiento del secreto: "A la mujer y en la carta, lo que dirías en plaza", "Hombre poco discreto es el que a su mujer dice su secreto".

Una de las armas más potentes que utiliza la mujer para controlar al hombre son las lágrimas ("Lo que la mujer no consigue hablando, lo consigue Ilorando", "Mujer, Ilora y vencerás") y la astucia ("La mujer y la raposa, astutas y engañosas", o "La más cauta es tenida por más casta").

Se defiende una diferencia que debe reconocerse entre hombres y mujeres: "Las mujeres nunca son como los hombres, sino mejores o peores" y "Donde hay mujeres, hay pesares y placeres". Pero parecen necesarias para la vida del hombre: "Hombre, sin mujer al lado, nunca bienaventurado", "La mujer es la mitad del hombre", "Rubias y morenas, sacan a los hombres de penas" o"Sin una mujer al lado, el hombre es un desdichado". Y con capacidad de 
mando, ordenación y decisión: “Casa sin mujer, gente sin capitán" y que si desaparece, fracasa el hogar: "Mujer muerta, casa deshecha".

Posiblemente por esa relevancia, existen asimismo muchos ejemplos de devaluación de la mujer, porque "La mujer es un mal necesario", "La mujer, como la vejez, un mal deseado es". Y puede resultar en peligros y sinsabores: "La mujer que sola piensa, mal piensa" y debe tener una función cuando sea necesario ("Las bromas y la mujer, cuando sean menester", o "La cara bonita y la intención maldita"), necesitan instrucción ("La mujer y el potro, que los dome otro").

Desde aquí, existen un número de refranes que pueden ser calificados directamente como machistas (como "La mujer en el hogar, sin salir ni a trabajar", o "A la moza y al fraile, que no les dé el aire") y otros en los que se defiende la bondad de la mujer: su recato, saber estar, virtuosa, hermosa y potenciadora de la acción del marido. Y asimismo, se advierte de los peligros que conlleva el tratarla mal o dejarla que maneje todas las situaciones, en cuyo caso, el hombre se presenta como un desdichado (sirva como ejemplo: "De la mujer, mucho bueno has de esperar y mucho malo has de temer").

Representan, además, una fuente de consejos. Se valora el primer consejo que se pida ante un problema ("De la mujer, el primer consejo; el segundo no le quiero"; "De la mujer, el consejo repentino; del hombre, el meditado y detenido"), pero no los siguientes, posiblemente, por la atribución de mayor intuición y rapidez en la percepción de los problemas interpersonales en las mujeres que en los hombres aunque tienden a ser valiosos ("El conse- jo de la mujer es poco, y el que no lo toma es loco"). Asimismo, en el caso de ancianos, se valora muy mucho "la conseja" de las viejas y no hacer caso de ello es imprudencia ("Del viejo, el consejo; de la vieja, la conseja").

Sin embargo, los papeles que se atribuyen a la mujer son los propios de la sociedad tradicional de ocupación en las tareas de hogar, gestación y crianza de hijos y la atribución, casi en exclusiva del mundo sentimental positivo en cuanto a expresión emocional ("La mujer es la salud y la calamidad de la casa") y necesarias para el mantenimiento de la casa ("Las mujeres, donde están, sobran; y donde no están, hacen falta").

El destino previsto para la mujer, es en el refranero, el matrimonio y manejo del marido y del hogar ("Marido rico y necio, no tiene precio") y, en el establecimiento de relaciones emocionales, hay que disimular información al pretendiente con toda intención ("El novio, no vió, porque si viera, novio no fuera").

En general, se le da mucha importancia al "saber vivir" de las mujeres y sus capacidades para controlar las situaciones de relaciones interpersonales $y$, en especial, las relaciones con los hombres, que aparecen más como débiles ante las cualidades de manejo de situaciones que desarrollan las mujeres, cualidades que no proceden de una educación reglada. Mucho se ha hablado del machismo presente en el refranero y el autor de estas líneas cree que más que machismo por devaluación se trata de una manera de esconder el respeto y hasta el miedo y el temor ante las múltiples armas y recursos que se le atribuye a la mujer para interactuar con el varón. 


\section{Conclusiones y discusión}

El principal objetivo que poseen estas páginas es el de mostrar la potencialidad del refranero para ofrecer una imagen comprensible de lo que se ha venido en Ilamar la "teoría lega" de la sabiduría y que representa una alternativa a la ofrecida por los estudios realizados hasta el momento. Esta versión representa más que un modelo acabado, unos apuntes que permiten crear instrumentos y recoger información empírica contemporánea respecto a los modos de entender tradicional y culturalmente la sabiduría en nuestro país. Y pensamos que ese objetivo se ha logrado.

La sabiduría se presenta como un atributo de excelencia humana, cercana a la inteligencia en el caso de adultos, aunque no representa la perfección en la medida en que, impide su existencia contemporánea con el afecto positivo y en general, con el amor. Pueden diferenciarse unas dimensiones racionales que caracterizan este "saber":

(i). Capacidades cognitivas de análisis de la realidad socio-personal (en la que la mujer se reconoce como más eficaz en la medida en que ella domina al hombre) y que se confunden con la inteligencia en sentido popular.

(ii). Capacidad de pensamiento y actuación ética (la "conciencia" unida a la "ciencia") a la que se da tanta importancia como a las capacidades cognitivas de análisis de la realidad. Aquí desempeña un papel importante la prudencia.

(iii). Capacidad y realización de acción directa (hacer cosas) o indirecta (dar consejo) además de conocer y leer. Por ello es un atributo más complejo que lo meramente cognitivo. (iv). El pensamiento y la acción sabia incluyen de maneras diversas componentes de necedad de manera que la conducta del sabio es una mezcla de sabiduría y estupidez.

Se anotan, además, algunas características específicas como (a) la relación entre sabios y necios, cuando existe, no es cordial puesto que representan modos de pensar y hacer incompatibles en la mayoría de las ocasiones; (b) mientras la necedad suele ir acompañada de bienestar económico, la sabiduría se sintoniza más con la pobreza; (c) de todas las sabidurías, la que se refiere al conocimiento y aplicación al mundo intrapersonal del propio sabio es la más difícil; (d) vejez y sabiduría poseen significaciones distintas y no hay ninguna garantía de que el anciano sea más sabio que anciano;

(v). Existen unos atributos psicológicos negativos que se encuentran presentes en los sabios y entre ellos cabe distinguir el temor (fobias, ansiedad), la avaricia, la escasa suerte en el amor, la incapacidad de amar y pesares y sufrimientos, junto con la infelicidad en general.

Por lo que se refiere al papel asignado y calificado para la mujer, en el discurso hay un predominio general de sabiduría atribuido a hombres frente a mujeres. De la mujer se tiene una visión dicotómica y dubitativa, atribuyéndole una mayor competencia en relaciones personales (domina al marido y mantiene el orden de la casa de manera que su desaparición lleva consigo la destrucción de la familia). Y a la vez, aparece como un personaje necesario y temido (si se le agravia llega ser peor que el demonio) y posee una mayor facilidad de expresión emocional ("La mujer ríe cuando puede y llora cuando quiere"), nadie (hombres y mujeres) 
debe fiarse de las lágrimas de una mujer. Pero no solamente domina esta parcela de una teórica inteligencia emocional, sino además, reconociendo su necesidad, asimismo el peligro de su dominio ("La mujer, si es hermosa, te la pegará; si es fea te cansará; si pobre, te arruinará, y si rica, te gobernará"), puesto que hasta posee un control notable sobre su enfermedad, si padece alguna ("Mujer doliente, mujer para siempre"). Es en este tipo de reflexiones y consideraciones en el que el autor de estas páginas tiene para sí que el "machismo" tan denostado y vituperado que dicen que se encuentra presente en el refranero español, lo está, pero de la misma manera, existen dimensiones, atributos y manera de entender a la mujer respaldadas por un respeto y hasta temor reverencial hacia sus maneras de ver la realidad y de actuar sobre ella. El universo de la mujer, es verdad que se restringe al hogar, pero en él, el hombre nada puede; ni siquiera limitar el poder que ejerce la mujer dentro de una concepción, muy posiblemente excesivamente tradicional y muy alejada de la competencia que ha demostrado ya la mujer con toda razón en las diversas áreas de funcionamiento social y laboral en la sociedad contemporánea. Pero pensamos que deben ser tomadas en consideración ambas líneas de pensamiento y no solamente una de ellas.

Un elemento a tener en cuenta y que no aparece en los resultados publicados sobre teorías legas de sabiduría es que, posiblemente por la metodología utilizada, las características y atributos que se incluyen son todos positivos de manera que se podría identificar al sabio como el ser humano que es un dechado de perfecciones sin mácula alguna (tanto en la bibliografía europea como en la americana). EI refranero acerca el sabio a una "humanidad" realista en la medida en que, como ser humano, se detectan insuficiencias y problemas que le hacen, posiblemente, más humano. $Y$ a partir de estas caracterizaciones, la recomendación para seguir trabajando en sabiduría consistiría en incluir estos tipos de atributos, asimismo, entre el material a calificar y exigir un conocimiento lo más profundo posible, de las personas "sabias" a calificar.

\section{Referencias}

Ardelt, M. (2003). Development and empirical validation of a three-dimensional wisdom scale, Research and Aging, 25, 275-324.

Bassett, C. (2011). Understanding and teaching practical wisdom, New Directions for Adult and Continuing Education, 131, 35-44.

Baltes, P. B. (2004). Mind as orchestration of mind and virtue, Berlín, mimeo.

Baltes, P. B. y Staudinger, U. M. (1985). Manual of the Berlin Paradigm in Wisdom, Berlín, mimeo.

Brent, S.B. y Watson, D. (1980). Aging and wisdom: individual and collective aspects, San Francisco, Meeting Gerontological Society Americain.

Clayton, V. P. (1976). A multidimensional scaling analysis pf the concept of wisdom, Ph.D. no publicada, University of Southern California.

Clayton V. P. y Birren,J. E. (1980). The development of wisdom across the life-span: A re-examination of an ancient topic. En P. B. Baltes y O.G. Grim (Eds.), Life-span development and behavior, vol.3, 103-135, New York: Academic Press.

Correas, G. (1627/1992). Vocabulario de refranes y frases proverbiales y otras fórmulas comunes de la lengua castellana, en que van todos los impresos antes y otra gran copia que junto el Maestro Gonzalo Correas, Catedrático de Griego y Hebreo en la Universidad 
de Salamanca. Van añadidas las declaraciones y aplicación adonde parecio ser necesaria. Al cabo se ponen las frases más llenas y copiosas, Lyon, Institut d'Études Ibériques et Ibéro-Americaines de l'Université de Bordeaux; reedic., Madrid: Visor libros.

Erikson, E. H.(1959). Identity in the life cycle. Psychological issues, New York: International University Press.

Erikson, E. H. (1982). The life cycle completed, New York: W.W. Norton.

Erikson, E. H., Erikson, J. M. y Kivnick, H.Q. (1986). Vital involvement in old age, New York: W.W. Norton.

Holliday, S.G. y Chandler, M.J. (1986). Wisdom: Explorations in adult competence, Basel: Karger.

Iribarren, J. M. (1994). El porqué de los dichos. Sentido, origen y anécdota de los dichos, modismos y frases proverbiales de España y otras muchas curiosidades, Sexta edición, Pamplona: Gobierno de Navarra.

Martínez Kleiser, L. (1989). Refranero General Ideológico Español, Madrid: Hernando.

Orwoll, L. y Perlmutter, M. (1990). The study of wise persons: personality perspective. En R.J. Sternberg (Ed.). Wisdom. Its nature, origins and development (pp. 160-177). New York: Cambridge University Press, 160-177.

Pelechano, V. y González, P. (2004). La concepción lega de la sabiduría, inteligencia y envejecimiento en adolescentes, Análisis y Modificación de Conducta, 30, 437-460.

Pelechano, V. y González, P. (2006). Teoría lega de sabiduría en adultos y relaciones con el yo ideal, Análisis y Modificación de Conducta, 32, 65-84.

Perlmutter, M., Adams, C., Nyquist, L. y Kaplan, C. (1988). Beliefs about wisdom; Trabajo no publicado, citado por Orwoll y Perlmutter (1990).

Rodriguez Marín, F. (1926). Más de 21000 Refranes Castellanos no contenidos en la copiosa colección del Maestro Gonzalo Correas, Madrid, Tipografía de la Revista de Archivos, Bibliotecas y Museos.

Sbarbi, J.M. (1891/1980). Monografía sobre los refranes, adagios y proverbios castellanos. Madrid, Atlas. 
